\title{
Projeto de Extensão em uma Escola de Pacientes Especiais: relato de experiência
}

\section{Juliane Bervian}

Universidade de Passo Fundo | Brasil jbervian@hotmail.com

\section{Stefano Reusch Cunha}

Universidade de Passo Fundo | Brasil stefano.cunha@gmail.com

\section{Camila da Costa Matsdolfo}

Universidade de Passo Fundo | Brasil ccmatsdolfo@yahoo.com.br

\section{Resumo}

Este texto apresenta um relato de experiência de um Projeto de Extensão que possui o objetivo de promover ações educativas e preventivas aos portadores de necessidades especiais, bem como intervenções curativas possíveis no ambiente da escola. Este Projeto foi proposto pela existência de um ambulatório odontológico no local, embora, além disso, os acadêmicos do curso de Odontologia e Fonoaudiologia realizem atividades diversificadas na escola. As experiências relatadas contribuem para aumentar a interação entre os grupos, salientar a prevenção e motivar para o autocuidado, qualificando os acadêmicos que se inserem em atividades diferentes das propostas no ensino convencional da graduação.

\section{Palavras-chave}

Pessoas com Deficiência; Criança Excepcional; Equipe de Assistência ao Paciente. 


\section{Introdução}

De acordo com a Organização Mundial da Saúde (OMS), quase um quinto do total da população global, isto é, mais de um bilhão de pessoas em todo o mundo apresentam algum tipo de deficiência ou dificuldades significativas (OMS, 2012). No Brasil, o Instituto Brasileiro de Geografia e Estatística (IBGE) estimou no ano de 2010, quase 24\% da população, ou seja, cerca de 45 milhões de brasileiros nessa condição (IBGE, 2010).

Paciente com necessidades especiais é aquele indivíduo que apresenta qualquer tipo de condição que o faça necessitar de atenção diferenciada por um período de sua vida ou indefinidamente. 0 portador de deficiência, seja ela física ou mental, encontra-se em uma condição de dependência. Os cuidados necessários podem variar dependendo do tipo e do grau da deficiência. Dessa maneira, a sua delimitação é crucial para determinar a melhor forma de intervenção (CABRAL; MORAES; SANTOS, 2003; CABRAL et al. 2004).

As abordagens multidisciplinares no atendimento de pacientes especiais passam por inúmeras demandas, sejam elas de cuidados médicos, odontológicos, nutricionais, fonoaudiológicos, entre outros, de caráter não apenas terapêutico, mas também, e principalmente, preventivo. Para isso, tanto os profissionais como as instituições que acolhem esses pacientes devem estar qualificados para suprimir ou minimizar as implicações da deficiência e, ainda, fornecer condições a uma melhor qualidade de vida (RESENDE et al. 2005).

O tratamento odontológico de pacientes com deficiência envolve a compreensão das dificuldades específicas (dificuldades motoras, dificuldades devido à falta de comunicação, necessidades odontológicas acumuladas, graus de limitação física, dentre outras) e inespecíficas (falta de profissionais habilitados, barreiras arquitetônicas e a superproteção da criança com deficiência) que envolvem o tratamento (GUIMARÃES; AZEVEDO; SOLANO, 2006). Além disso, é essencial que haja o envolvimento e o comprometimento dos pais/responsáveis no planejamento das atividades, juntamente com a equipe multidisciplinar na tentativa de minimizar a possibilidade de intervenções futuras (HADDAD, 2007).

Esses pacientes, que apresentam tais limitações, de ordem intelectual ou física, fazem parte dos grupos que requerem a instalação de programas preventivos de 
excelência (PEREIRA et al. 2010). A educação dos pacientes e seus pais ou cuidadores visando à prevenção deve, então, ser planejada desde os estágios mais precoces, pois minimiza a doença e as intervenções curativas (SPECTOR et al. 2003). As medidas deverão ser estabelecidas principalmente através da motivação da família e da população alvo.

Há profissionais que atuam com esses grupos, portanto, salienta-se a importância do trabalho como instrumento fundamental no atendimento das complexidades da promoção e da educação em saúde nas comunidades. 0 trabalho, que utiliza a estratégia de formação de grupos, tem a vantagem de fomentar a produção coletiva do conhecimento, bem como a reflexão sobre a realidade vivenciada por seus membros. 0 processo reflexivo se mostra importante na medida em que possibilita a construção de estratégias de enfrentamento dos desafios que, por vezes, permeiam suas situações de vida (SOUZA et al. 2005). Em função disso, este trabalho relata uma experiência de intervenção preventiva e terapêutica de maneira interdisciplinar nos alunos da Associação de Pais e Amigos dos Excepcionais (APAE) de Passo Fundo-RS.

\section{Metodologia}

Este relato descreve a experiência de um Projeto de Extensão denominado "Atenção odontológica integral e interdisciplinar em pacientes com necessidades especiais na APAE de Passo Fundo - RS". O mesmo foi criado e institucionalizado pela Universidade de Passo Fundo (UPF) por meio de uma parceria entre os cursos de Odontologia, Fonoaudiologia e a APAE.

Desde agosto de 2013 as atividades são realizadas semanalmente na escola APAE, respeitando o calendário acadêmico dos docentes e discentes da Universidade. Todos os alunos da escola estão incluídos, independentemente da população ser muito heterogênea, em função dos diversos tipos de necessidades especiais, dentre as quais encontram-se os indivíduos portadores de Síndrome de Down, Encefalopatia Crônica não Progressiva, Síndrome de Pierre Robin, Deficiência Mental, Autismo, entre outros.

A cada semestre, novas atividades são programadas, isso produz uma possibilidade de crescimento no grupo e de avaliações de erros e acertos. Durante o ano 
de 2015, além de uma professora do Curso de Odontologia, uma professora do Curso de Fonoaudiologia e os acadêmicos de ambos os cursos participaram das atividades do projeto. Na busca de melhorar as condições de saúde bucal dessa população, a odontologia possui uma visão mais resolutiva e imediata, embora preventiva da adequação e integridade das estruturas bucais, enquanto a fonoaudiologia visa habilitar ou reabilitar as funções do Sistema Estomatognático (SE). Dessa forma, ambos os cursos trabalham em conjunto para restabelecer o equilíbrio miofuncional destas funções, e as ações em conjunto refletem uma preocupação que soma ao ensino e a extensão fora do ambiente acadêmico.

\subsection{Organização das atividades}

Desde a criação do projeto com as atividades iniciais até os dias atuais, várias adaptações foram realizadas ao projeto original. As atividades programadas são discutidas em grupo, portanto em cada semestre ocorre troca dos profissionais. Os profissionais que atuam na Escola APAE também auxiliam de forma a supervisionar os alunos e adaptar questões teóricas acadêmicas com atividades práticas do meio e da problemática do ambiente.

As atividades realizadas neste Projeto de Extensão consistem em oficinas intercaladas (odontológica e fonoaudiológica), nas quais os alunos da escola participam realizando as tarefas propostas pelos acadêmicos e sendo estimulados para alcançar resultados cada vez mais significativos. As oficinas são realizadas por turma, e por algum período a rotina da aula é substituída pela atividade programada naquela semana.

A oficina odontológica trabalha as questões de orientação na escovação além de orientar a prática da utilização de dentifrício fluoretado (pastas ou cremes dentais). Através de uma forma mais lúdica também são feitas atividades de pintura em desenhos de dentes, enxaguantes bucais, escovas, cremes e fios dentais para conscientizar ainda mais a importância do uso desses itens para a manutenção da saúde bucal. Os acadêmicos de Odontologia interagem com os profissionais da Terapia Ocupacional e viabilizam as questões de motricidade e apreensão da escova dentária. Os atendimentos odontológicos de forma convencional, no consultório odontológico também são ofertados por este projeto e ocorrem conforme a demanda de agendamento. 
Na oficina fonoaudiológica, para adequar as estruturas correspondentes à área da Motricidade Orofacial (MO), bem como as funções do SE, são realizados exercícios de fácil execução e para a prática de grupo. As oficinas trabalham os estímulos aromáticos e gustativos utilizando pedaços de frutas (banana, bergamota, morango, melão ou laranja), em alguns momentos com olhos vendados para estimular a percepção olfatória, em outros momentos não. A mastigação é trabalhada também, no padrão bilateral alternado, sempre que possível para o correto emprego da articulação temporomandibular e a adequada formação do bolo alimentar, exercitando a musculatura dos órgãos fonoarticulatórios. É incentivado o trabalho para habilidade do sopro através de um canudo em um copo d'água, com apito e com bolinhas de sabão, ambos para controle da coordenação da função respiratória. Também são realizadas atividades de estalo de língua, bico-sorriso e sucção de bochechas para trabalhar a mobilidade de língua, lábios e bochechas, respectivamente. Ao término das atividades fonoaudiológicas, os sujeitos são bonificados com adesivos, ou alguma outra possibilidade de brindes.

O trabalho em conjunto da Odontologia e da Fonoaudiologia na MO, neste projeto, visa melhorar os elementos e as funções do SE, pois ambas se completam e uma depende da outra na busca pela saúde integral do paciente. Tais profissões são realmente aliadas uma da outra, nesta proposta que visa melhorar a qualidade de vida do paciente. No entanto, a interação dos acadêmicos ocorre em áreas inesperadas, pois conforme a necessidade eminente o grupo busca e discute soluções possíveis.

\subsection{Questões éticas}

Este projeto foi enviado ao Comitê de Ética em Pesquisa da Universidade de Passo Fundo (http://aplicacao.saude.gov.br/plataformabrasil) e aprovado pelo parecer CAAE: 43050515.8 .0000 .5342$.

Os pais e responsáveis dos alunos da APAE quando matriculam seus filhos recebem um Termo de Consentimento Livre e Esclarecido, explicando as atividades realizadas na escola e autorizando seu filho a participar do projeto. 


\section{Resultados}

Os resultados deste Projeto de Extensão são avaliados anualmente. Fazem parte do relatório que a Instituição indica como avaliador de renovação para o ano seguinte. De acordo com os relatórios, aproximadamente 200 alunos da escola APAE são beneficiados anualmente, sendo que 120 deles através de atendimentos individuais no consultório odontológico e cerca de aproximadamente 80 deles nas oficinas que ocorreram durante o período. Além disso, são realizadas palestras, uma delas aos pais e cuidadores e outra delas aos alunos da escola.

Em relação à academia, por ano, até 2 docentes estão envolvidos neste trabalho e aproximadamente 10 alunos de graduação. Sendo que os alunos mudam de um semestre para outro. Um ou dois desses acadêmicos recebem bolsa de incentivo pela participação de Projeto de Extensão (Paidex - anual), e os outros alunos são voluntário. 0 projeto originou comunicações em eventos locais de tipos variados, duas apresentações em Semana Acadêmica e uma apresentação em Mostra do Conhecimento. Além de ter incentivado um Trabalho de Conclusão de Curso de pesquisa sobre pacientes com Síndrome de Down.

Embora esses números sejam satisfatórios e importantes, algumas questões a seguir são tão ou mais relevantes neste projeto. A participação do grupo se identificou e promoveu a construção do saber neste ambiente, a troca entre a academia e a instituição que se adapta com a periodicidade dos acadêmicos, bem como a novidade das atividades é impressionante. Nesses últimos semestres, a introdução de práticas voltadas ao grupo e maior participação na vida da escola trouxe experiências agregadoras a todos. A inserção do acadêmico em um ambiente externo aos muros da faculdade e, neste caso, a um local diferenciado, torna o aprendizado consistente e eterno.

A participação da Escola, avaliando a possibilidade de renovação do projeto e demonstrando interesse na continuidade das atividades, auxilia mostrando os caminhos que ainda precisam ser trilhados, e apontando-nos alguns que já foram alcançados. 


\section{Discussão}

A Constituição Brasileira de 1988 já apresentava princípios gerais de políticas de inclusão das pessoas com deficiência. A partir daí, leis complementares relativas a aspectos educacionais, de acessibilidade, e de incentivo a empregos, vem sendo implementadas. Este processo, entretanto, tem sido bastante irregular: com avanços em alguns setores e lentidão em outros.

Na APAE/Passo Fundo existem 399 alunos que frequentam a instituição em turnos variados, alguns de manhã e outros à tarde, sendo que alguns alunos também frequentam as escolas da cidade. 0 sistema de inclusão identifica os alunos com menor comprometimento intelectual e auxilia a introdução dos mesmos nas escolas da rede pública do município. Para esses, as atividades da escola APAE são realizadas em turno inverso ao das atividades acadêmicas da escola convencional. Aos outros, com maior comprometimento, a escola APAE fica à disposição em horário integral.

A sociedade se preocupa muito com a incapacidade desses indivíduos, porém esquece que eles também precisam de educação e atendimentos especiais (SILVA; CRUZ, 2009).

Quando se trata de crianças com necessidades especiais, as questões relacionadas à interação social se tornam ainda mais importantes, já que, por possuírem algumas limitações, por exemplo, quanto às possibilidades de locomoção e exploração de objetos, são frequentemente consideradas incapazes de participar e contribuir nas atividades em grupo (SOUZA; BATISTA, 2008). Apesar das crianças com necessidades especiais apresentarem tais limitações, percebe-se, na maioria, o esforço para a realização das atividades. Isso pode ser incentivado pelo grupo familiar, mas normalmente é realizado pelo grupo escolar, que não discrimina e auxilia respeitando as individualidades. Mostrando e comprovando a importância da motivação na interação social e, particularmente, da interação entre os elementos de um grupo (CARVALHO; PEDROSA, 1989; CARVALHO; BERALDO, 1989). Isso nos remete à interação social que passa a ser vista como constitutiva do desenvolvimento cognitivo e da interação com o mundo físico (PEDROSA; CARVALHO, 1995).

Durante os trabalhos em grupos, desenvolvidos neste projeto de Extensão na APAE, tanto nas oficinas odontológicas quanto nas fonoaudiológicas, são empregadas, de 
maneira geral, abordagens favoráveis à conscientização dos indivíduos portadores de necessidades especiais. Sendo essas condutas lúdicas com reforço positivo e com atenção as formas de expressão, gestos e reações, para que os efeitos das oficinas sejam progressivos e gradativos ao longo das intervenções (SCHMIDT, 2001).

As oficinas presam pela prevenção da higiene bucal e readequação das estruturas oromiofuncionais, para que todos compreendam a complexidade do assunto, reforçando a necessidade de ter os corretos hábitos bucais e o funcionamento adequado das estruturas do SE. Conforme ressaltado na literatura, um dos mediadores do processo ensino-aprendizagem é a atividade lúdica. Nesse sentido, verifica-se que o lúdico contempla os critérios para uma aprendizagem efetiva, pois chama a atenção para um determinado assunto (COSCRATO; PINA; MELLO, 2010). Os alunos da APAE apresentam bastante entusiasmo durante as atividades e demonstram que as oficinas lhe dão mais conhecimento sobre estruturas e funções do próprio corpo. E a cada acerto deles vemos que isso estimula-os para melhorar.

Mesmo assim, durante o processo de intervenção, as pessoas que se propõem a um trabalho assim precisam estar capacitadas, orientadas e, no caso dos alunos, em supervisão constante. 0 trabalho com pacientes especiais em um ambiente escolar como a APAE não impede que circunstâncias inevitáveis aconteçam, desde a mais leve, onde o aluno se nega a colaborar, até aquelas que envolvem uma reação comportamental mais grave e/ou agressiva. Evidentemente, em qualquer um dos casos, é indispensável o conhecimento do modo de agir e a rapidez na ação, de maneira racional e ética, para ir além dos empecilhos e manter a busca pelo progresso do trabalho (BOTAZZO, 2005).

Ao longo do projeto, duas características foram incentivadas e merecem destaque: a interdisciplinaridade e o foco preventivo. A interdisciplinaridade por meio da integração das áreas da saúde, distintas, trabalhando entre si e trocando conhecimentos para melhor atender as necessidades dos indivíduos (TORRES, 1998); e o foco preventivo deu-se através das oficinas com práticas odontológicas e fonoaudiológicas para intervir justamente nos aspectos que favorecem o desequilíbrio oromiofuncional e manter ou melhorar a qualidade e o uso adequado das estruturas trabalhadas.

Sendo assim, este projeto compreende os fatores essenciais para que ocorra o cuidado integral do paciente, por meio da interação interdisciplinar e do foco 
preventivo, assim, o formato adotado neste projeto, ao contrário do foco unicamente curativo, visa o atendimento do paciente como um todo e, assim, contemplar a saúde integral (DORO et al. 2006).

A questão da integralidade, que seria a de compreender o paciente e propor atendê-lo por inteiro em suas necessidades de saúde, é evidente e de suma importância no atendimento às pessoas com necessidades especiais. A prática clínica integral é desejável como ideal para a equipe de saúde bucal porque o trabalho do dentista e, por vezes, do fonoaudiólogo é fragmentado (BOTAZZO, 2005).

Desta forma, tais procedimentos e as atividades oferecidas em grupo e incentivando a automatização do cuidado, e também do autocuidado, retira os profissionais da frieza dos métodos em que o tecnicismo é produzido e reproduzido, permitindo que esse profissional seja habilitado a atender pessoas, respeitando limites, condições especiais e visando ao atendimento integral (BOTAZZO, 2005; FONSECA et al. 2010).

E, por fim, outro objetivo intrínseco em um projeto de extensão institucional é poder ofertar ao acadêmico uma vivência diferente, contextualizando com o futuro mercado de trabalho e precocemente inserindo-o na sociedade. Hoje em dia, cada vez mais sabe-se a importância de o aluno vivenciar na prática tudo o que é aprendido na teoria, expandindo seu conhecimento, agregando valor e experiência na vida acadêmica. Pois com o passar dos anos sentiu-se a necessidade de atuar de diferentes formas e o profissional deve estar preparado para essa etapa de sua carreira (COSCRATO; PINA; MELLO, 2010).

O conceito de educação em saúde também vai além da transmissão de informações, configurando combinações de experiências de aprendizagem delineadas com vistas a facilitar ações voluntárias conducentes à saúde. Essas combinações envolvem troca de experiências de vida, aspectos comportamentais, medidas terapêuticas e interacionais (COSCRATO; PINA; MELLO, 2010).

Visando isso, o projeto realizado na APAE proporciona um vasto conhecimento com experiências extraclasse e interdisciplinar, assim possibilitando vivenciar na prática aspectos que nem sempre são relatadas na teoria ou em livros. E também tornando o acadêmico cada vez mais confiante e mostrando que o trabalho em grupo, muitas vezes, traz mais e melhores resultados. A interdisciplinaridade é fundamentalmente um 
processo e uma filosofia de trabalho que entra em ação na hora de enfrentar os problemas e questões que preocupam cada sociedade (TORRES, 1998), formando um profissional mais capacitado, preparado e qualificado para lidar com possíveis adversidades futuras.

A realidade humana é, por um lado, uma realidade biológica e, por outro lado, uma realidade auto-biológica. Quer dizer, uma realidade mitológica onde se crê que possa existir um padrão normal (SILVA; PANHOCA; BLACHMAN, 2004). Resta saber se somos exclusivos e únicos, e absolutamente normais para efetuarmos este tipo de conceito.

\section{Considerações finais}

Este projeto é uma experiência válida para todos os envolvidos, pois dificilmente encontrariam uma oportunidade de aprimorar e trocar conhecimentos senão em um ambiente onde à demanda de pacientes requer cuidados além dos padrões de normalidade. Os resultados mostraram que o número de indivíduos beneficiados com este formato de intervenção, integral e preventiva, aumentou consideravelmente em um curto espaço de tempo. E muito embora os números e as quantidades não determinam prioridade neste projeto, a forma como foi alcançada sim. A possibilidade do atendimento curativo e a alternância com as oficinas proporciona ao paciente com necessidade especial uma melhor compreensão da possibilidade do autocuidado.

\section{Referências}

BOTAZZO, Carlos. Abordagens em saúde bucal. A questão da integralidade. In: GARCIA, Danielli (Org.). Novos rumos da saúde bucal: os caminhos da integralidade. Rio de Janeiro; ABO/ANS/UNESCO, 2005, p. 43-47.

CABRAL, Ivone Evagelista; MORAES, Juliana Rezende; SANTOS, Flavia Fragoso. O egresso da terapia intensiva neonatal de três instituições públicas e a demanda de cuidados especiais. Escola Anna Nery. Revista de Enfermagem, v.7, n.2, p.211-218, 2003.

CABRAL, Ivone Evangelista; SILVA, Jaqueline de Jesus; ZILLMANN, Danielli de Oliveira; MORAES, Juliana Rezende; RODRIGUES, Elisa da Conceição. A criança egressa da terapia intensiva na luta pela sobrevida. Revista Brasileira de Enfermagem, v.57, n.1, p.35-39, 2004. 
CARVALHO, Ana Maria Almeida; PEDROSA, Maria Isabel. Brincar juntos: natureza e função da interação de crianças. São Paulo: EDICON/EDUSP, 1989.

CARVALHO, Ana Maria Almeida; BERALDO, Katharina Elisabeth Arnold. Interação criança-criança: o ressurgimento de uma área de pesquisa e suas perspectivas. Cadernos de Pesquisa, São Paulo, n. 71, p. 55-61, 1989.

COSCRATO, Gisele; PINA, Juliana Coelho; MELLO, Debora Falleiros. Utilização de atividades lúdicas na educação em saúde: uma revisão integrativa da literatura. Acta Paulista Enfermagem, São Paulo, v. 23, n. 2, p. 257-263, 2010.

DORO, Guilhermo Manfi; FIALHO Lucas Meneghello; LOSEKANN Maximiliano; PFEIFF Dênis Nestor. Projeto “Odontologia Hospitalar”. Revista Abeno, v.6, n.1, p. 49-53, 2006.

FONSECA, Alexandre Luiz Affonso; AZZALIS, Ligia Ajaime; FONSECA, Fernando Luiz Affonso; BOTAZZO Carlos. Análise qualitativa das percepções de cirurgiões-dentistas envolvidos nos atendimentos de pacientes com necessidades especiais de serviços públicos municipais. Revista Brasileira Crescimento Desenvolvimento Humano, v.20, n.2, p. 208-216, 2010.

GUIMARÃES, Amanda Oliveira; AZEVEDO, Isabelita Duarte; SOLANO, Maria da Conceição Pereira Pinto. Medidas preventivas em Odontologia para pacientes portadores de necessidades especiais. Revista Ibero-Americana de Odontopediatria \& Odontologia do Bebê, v.9, n.47, p.79-84, 2006.

HADDAD, Aida Sabbagh. Odontologia para pacientes portadores de necessidades especiais. São Paulo: Santos; 2007.

INSTITUTO BRASILEIRO DE GEOGRAFIA E ESTATÍSTICA. IBGE. Censo Demográfico 2010. Disponível em: <http://www. censo2010.ibge.gov.br>.

ORGANIZAÇÃO MUNDIAL DA SAÚDE. OMS. The World Bank. Relatório Mundial sobre a Deficiência. Trad Lexicus Serviços Linguísticos - São Paulo. SEDPcD: São Paulo, 2012.

PEDROSA, Maria Isabel; CARVALHO, Ana Maria Almeida. A interação social e a construção da brincadeira. Caderno de Pesquisa, São Paulo, n.93, p. 60-65, 1995.

PEREIRA, Luciana Macedo; MARDERO Etiene; FERREIRA, Simone Helena; KRAMER, Paulo Floriani; COGO, Rafael Barreto. Atenção odontológica em pacientes com deficiência: a experiência do curso de Odontologia da ULBRA Canoas/RS. Stomatos, Canoas, v.16, n.31, p. 92-99, 2010.

RESENDE, Vera Lucia Silva; CASTILHO, Lia Silva de; VIEGAS, Claudia Maria de Souza; SOARES, Maria Alice. Atendimento odontológico a pacientes com necessidades especiais. In: 8o Encontro de Extensão da UFMG: 2005. Belo Horizonte. Anais do 8o Encontro de Extensão da UFMG; 2005. p. 1-6. 
SCHMIDT, Rosangela Maria. Pacientes especiais: portadores de deficiências neuropsicomotoras. In: CORRÊA, Maria Salete Nahás Pires. Odontopediatria na primeira infância. São Paulo: Santos; 2001.

SILVA, Luiz Candido Pinto; CRUZ, Roberval de Almeida. Odontologia para pacientes com necessidades especiais. Protocolos para o atendimento clínico. São Paulo: Santos, 2009.

SILVA, Olga Maria Panhoca da; PANHOCA, Luiz; BLACHMAN, Isaac Tobias. Os pacientes portadores de necessidades especiais: revisando os conceitos de incapacidade, deficiência e desvantagem. Salusvita, Bauru, v.23, n.1, p.109-116, 2004.

SOUZA, Aline Corrêa de; COLOMÉ, Isabel Cristina dos Santos; COSTA, Lilian Escopelli Deves; OLIVEIRA, Dora Lucia Lendes Corrêa. A educação em saúde com grupos na comunidade: uma estratégia facilitadora da promoção da saúde. Revista Gaúcha Enfermagem, v. 26, n. 2, p. 147 - 153, 2005.

SOUZA, Carolina Molina Lucenti de; BATISTA, Cecília Guarnieri. Interação entre crianças com necessidades especiais em contexto lúdico: possibilidades de desenvolvimento. Psicologia, Reflexão e Crítica, Porto Alegre, v. 21, n. 3, p. 383-391, 2008 .

SPECTOR, Aimee; THORGRIMSEN, Lene; WOODS, Bob; ROYAN, Lindsay; DAVIES, Steve; BUTTERWORTH, Margaret; ORRELL, Martin. Efficacy of na evidence-based cognitive stimulation therapy programme for people with dementia: randomised controlled trial. British Journal of Psychiatry, v. 183, n.3, p.248-254, 2003.

TORRES, Santomé Jurjo. Globalização e interdisciplinaridade: o currículo integrado. Porto Alegre: Artmed; 1998. 
Extension Project at a School of Special Patients: experience report

\begin{abstract}
This text presents an Extension Project experience report that aims to promote educational and preventive actions for people with special needs, as well as possible curative interventions in the school environment. This project was proposed due to the existence of a dental outpatient clinic in the locality, although, in addition, the students of the course of Dentistry and Speech and Hearing Therapy carry out diversified activities in the school. The reported experiences contribute to increase the interaction between the groups, highlight the prevention and motivate for self-care, qualifying the students that are inserted in activities different from the proposals in the conventional teaching of the graduation.
\end{abstract}

\section{Keywords}

Disabled persons; Child exceptional; Patient care team

Original submetido em: 23 mar. 2016

Aceito para publicação em: 30 nov. 2017
Proyecto de Extensión en una Escuela de Pacientes Especiales: relato de experiência

\section{RESUMEN}

Este artículo presenta una cuenta de Extensión La experiencia del proyecto que tiene el objetivo de promover acciones educativas y preventivas para las personas con necesidades especiales, así como las posibles intervenciones curativas en el entorno escolar. Este proyecto fue propuesto por la existencia de una clínica dental en el lugar, sino también el curso académico de Odontología y Fonoaudiología realizar diversas actividades en la escuela. Experiencias reportadas contribuyen a aumentar la interacción entre los grupos, hacen hincapié en la prevención y motivar para el autocuidado, los estudiosos de calificación que caen en diferentes actividades propuestas en la graduación de la educación formal.

\section{Palabras clave}

Personas con discapacidad; Niño excepcional; Grupo de atención al paciente

Sobre o autores:

Juliane Bervian

Professora adjunta do Departamento de Ciências da Saúde, Universidade de Passo Fundo.

\section{Stefano Reusch Cunha}

Acadêmico de Fonoaudiologia, Universidade de Passo Fundo, bolsista de extensão Paidex.

\section{Camila da Costa Matsdolfo}

Acadêmica de Fonoaudiologia, Universidade de Passo Fundo, bolsista voluntária de extensão. 